Manuelle Medizin 2015 · 53:408

DOI 10.1007/s00337-015-0040-4

o Springer-Verlag

Berlin Heidelberg 2015

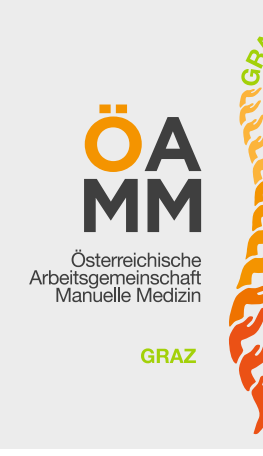

ÖAMM - Österreichische Arbeitsgemeinschaft Manuelle Medizin

Ausbildungsleiter:

Prof. Dr. H. Mengemann,

Univ. Prof. Dr. R. Lackner
Kärntner Straße 419, A-8054 Graz Tel. \& Fax.: +43 (0) 316283483

E-Mail: fortbildung@oamm-graz.at Web: www.oamm-graz.at

\title{
Kurse 2015
}

II. Kurse

04.12.-06.12.2015 Bad Häring

\section{Kurse}
12.12.-19.12.2015
Graz
KA1542

\section{Refresher Wochenende}

14.11.-15.11.2015 Loipersdorf

\section{Kurse 2016}

\section{Kurse}

13.02.2016-20.02.2016

07.05.2016-14.05.2016

11.08.2016-14.08.2016

18.08.2016-21.08.2016

15.10.2016-22.10.2016

\section{Kurse}

05.03.2016-12.03.2016

04.06.2016-11.06.2016

03.09.2016-10.09.2016

03.11.2016-06.11.2016

10.11.2016-13.11.2016

\section{Kurse}

10.03.2016-13.03.2016

18.03.2016-21.03.2016

18.06.2016-25.06.2016

17.09.2016-24.09.2016

26.11.2016-03.12.2016

\section{Kurse}

24.09.2016-01.10.2016

\section{Kurse}

09.04.2016-16.04.2016

10.12.2016-17.12.2016

\section{RE Woche}

14.03.2016-18.03.2016

\section{RE Wochenende}

12.11.2016-13.11.2016
KA1561

KA1641

(Kurs-Nr.)

KA1523 Teil 2

(Kurs-Nr.)

KA1611

KA1612

KA1613

KA1614

KA1621

KA1622

KA1623

KA1624

KA1631

KA1632

KA1633

KA1634

KA1651

KA1652

KA1671

\section{Ausbildungsziel}

Das Erlernen von klinisch-manuellen Untersuchungstechniken am Stütz- und Bewegungssystem zur therapeutischen Beeinflussung von reversiblen Funktionsstörungen.

Die Fortbildung dient damit der Erweiterung des Angebotes der kurativen, rehabilitativen und präventiven Medizin.

\section{Zielgruppe}

ÄrztInnen für Allgemeinmedizin und FachärztInnen aller Sonderfächer.

\section{Teilnahmevoraussetzung}

Frühest möglicher Beginn der Fortbildung ist nach der Promotion.

\section{Weiterbildungsdauer/Stundenerfordernis}

Mindestens 1,5 Jahre, nach den Richtlinien der Österreichischen Ärztekammer sind für das ÖÄK Diplom Manuelle Medizin insgesamt 300 Fortbildungsstunden (100 Stunden Theorie, 200 Stunden Praxis und Demonstrationen) erforderlich.

\section{Wesentliche Inhalte}

Erkennen und behandeln mit den Händen

Von der Arbeitshypothese zur Diagnose

Prävention

Rezidivprophylaxe

Terminänderungen/Absagen vorbehalten.

Wir weisen darauf hin, dass für die Kursteilnehmer der ÖAMM die Kurszeiten und Anwesenheitspflicht bindend sind!

Die Bestätigung der Fortbildungspunkte durch die österreichische Ärztekammer ist an die Erfüllung der Kursrichtlinien gebunden.

Verbindliche Anmeldungen und Auskünfte im Sekretariat der ÖAMM

E-Mail: fortbildung@oamm-graz.at

Internet: www.oamm-graz.at

Adresse: Kärntner Straße 419, 8054 Graz

Tel. \& Fax: +43 (0) 316-283 483 